\title{
Immediate Delivery versus Expectant Management in Pregnant Women with Preterm Premature Rupture of Membranes at 34 Weeks: A Cohort Study
}

\author{
Elsayed Elshamy, MD \\ Lecturer of Obstetrics and Gynecology, Faculty of Medicine. Menoufia University, Egypt \\ s_shamy77@yahoo.com,s_shamy77@hotmail.com
}

\begin{abstract}
:
Aim: to compare delivery to expectant management for cases of preterm premature rupture of membranes at 34 weeks.

Methods: This was a prospective study carried out at Menoufia University Hospital, Egypt on 84 pregnant women with preterm premature rupture of membranes at 34 weeks, the studied women were divided to 2 equal groups: group A managed by immediate delivery and group B managed expectantly for 2 weeks. Enrolled patients were followed to assess the maternal and neonatal outcome.

Results: There was higher cesarean section rate in group A ( $p<0.05)$., no significant differences between the 2 groups regarding puerperal pyrexia and postpartum hemorrhage, also, no significant differences related to maternal age and parity or basal total leucocytic count ( $p>0.05$ ) but follow up total leucocytic count was higher in group $B(p<0.05)$. Regarding neonatal outcome, group B had higher birthweight $(p<0.01)$, lower rate of TTN $(p<0.05)$ and neonatal hyperbilirubinemia $(p>0.05)$. No significant differences regarding Apgar score, neonatal intensive care admission and regarding respiratory distress syndrome only one case reported in group A but no statistical significance.
\end{abstract}

Conclusions: expectant management is wrranted for cases of recent preterm premature rupture of membranes at 34 weeks.

Keywords: expectant management, fetal outcome, maternal outcome, preterm premature rupture of membranes.

\section{INTRODUCTION}

Premature rupture of membranes or prelabour rupture of membranes means rupture of fetal membranes before onset of labour and the term preterm premature rupture of membranes means the occurrence of rupture of fetal membranes before term (before completed 37 weeks of gestation).One of its major sequelae is preterm delivery which is the leading cause of neonatal morbidity and mortality (about $40 \%$ of preterm births)[1]. Other sequelae include maternal and neonatal infection, placental abruption, cord compression and fetal deformities and lung hypoplasia if rupture of membranes occurs very early (before 26 weeks) $[2,3]$.

There is still many controversies regarding the optimal management of cases of preterm premature rupture of membranes and the item of controversies are much more in cases that occur before 34 weeks of gestation [4].

There is general agreement to conduct expectant management for cases of preterm premature rupture of membranes at gestational age less than 34 week so that to improve birthweight and to reduce the incidence of respiratory distress syndrome and in turn reducing neurological and developemental abnormalities[5,6]. Also the challenge against infection must run parallel to improving birth weight because it will lead to poor outcome and poorer outcome when associated with low birth weight baby [7].

In this study, we are interested in cases of preterm premature rupture of membranes presenting at 34 weeks of gestation evaluating whether the mother and the fetus can benefit from waiting for a reasonable time or not. 


\section{MATerials AND MethodS}

This prospective observational study was conducted at Obstetrics and Gynecology Department, Menoufia university hospital, Menoufia, Egypt in the period betweenJuly 2014 and March 2015.

The institutional review board approved the study protocol and an informed consent was obtained from all participants prior to commencing the study. For women agreeing to participate in the study, the following was done: Complete history taking, general and abdominal examination, local inspection using sterile speculum examination but no digital examination, Obstetric ultrasound and biophysical profile and complete blood count. Then after that initial basic evaluation we included women who met the following inclusion criteria in the study

\section{INCLUSION CRITERIA}

Pregnant women with singleton pregnancy at 34 weeks of gestation with preterm premature rupture of membranes diagnosed and evident clinically by sterile vaginal speculum examination

\section{EXCLUSION CRITERIA}

multiple pregnancy, the presence of uterine scar, parity of 3 or more, rupture of membranes for more than 2 days, vaginal bleeding, if the maximal vertical diameter of the largest pocket is less than $2 \mathrm{~cm}$, labour pains and clinical or laboratory evidence of chorioamnionitis.

Then women who were eligible and agreeing to participate in our study were randomly allocated using the computer system into 2 equal groups, group A and group B, for women in group A the following was done: hospital admission, antibiotic cover in the form of erythromycin then corticosteroid course in the form of 2 doses of $12 \mathrm{mg}$ dexamethasone intramuscular with 12 hours apart in between then we started induction of labour 24hours after the $2^{\text {nd }}$ dose of corticosteroids with careful follow up, CTG monitoring and partogramming to manage any abnormal progress or complications, after delivery neonatal evaluation, birthweight, Apgar score and careful follow up by the neonatologist in the nursery.

For women in group B: hospital admission, bed rest, sterile vulval pad, antibiotic cover in the form of erythromycin, complete blood count every other day, CTG daily then when the patient reaches 35 weeks, corticosteroid course in the form of 2 doses of $12 \mathrm{mg}$ dexamethasone intramuscular with 12 hours apart in between was given, then by the end of 35 weeks of gestation we started induction of labour, CTG monitoring and partogramming to manage any abnormal progress or complications, after delivery neonatal evaluation, birthweight, Apgar score and careful follow up by the neonatologist in the nursery.

For both groups follow up and antibiotic cover in the form of erythromycin $500 \mathrm{mg}$ taken orally every 6 hours continued until delivery or maximally for 10days (in group B)[8].

For both groups labour induction started by digital examination to assess Bishop score,if 6 or more induction was achieved by low dose syntocinon drip, if less than 6 , induction was achieved by misoprostol $25 \mathrm{ug}$ inserted vaginally in the posterior fornix and repeated every 6 hours with a maximum of 3 doses.

Outcome measures included maternal obstetric outcomes in the form of mode of delivery, postpartum hemorrhage, puerperal pyrexia and total leucocytic count and neonatal outcomes in the form of birthweight, Apgar score, respiratory distress syndrome, transient tachypnea of newborn (TTN), neonatal intensive care unit(NICU) admission and neonatal hyperbilirubinemia.

\section{Statistical Analysis}

Results were statistically analyzed by statistical package SPSS version 20(SPSS Inc., Chicago Ill). Student's t-test was used forquantitative data. Chi-Squared $\left(\chi^{2}\right)$ and Fisher's exact test were used for qualitative data. $\mathrm{P}<0.05$ was considered Significant

\section{ReSUlts}

Fig (1): flowchart.

Table (1): shows the characteristics of the studied women, where there was no statistically significant differences between the 2 groups regarding age, parity or basal total leucocytic count. 
Immediate Delivery Versus Expectant Management in Pregnant Women with Preterm Premature Rupture of Membranes at 34 Weeks: A Cohort Study

Table (2): shows the maternal obstetric outcome of the 2 groups where there was higher CS rate in group A $(28.6 \%)$ compared to (14.3\%) in group B but not reaching statistical significance, there was statistically significant difference regarding follow up total leucocytic count being higher in group B and there was no difference between the 2 groups regarding postpartum hemorrhage or puerperal pyrexia.

Table (3): shows the neonatal outcome of the studied women where there was highly significant difference in birthweight being higher in group B and this is logic, significantly higher incidence of transient tachypnea of newborn being higher in group A, neonatal hyperbilirubinemia was higher in group A and this is approaching, but not reaching, statistical significance. Otherwise there were no significant differences regarding Apgar score, respiratory distress syndrome (RDS) or NICU admission between the 2 groups.

Table1. Characteristics of the studied groups

\begin{tabular}{|c|c|c|c|c|c|c|}
\hline & \multicolumn{4}{|c|}{ Groups } & \multirow[b]{2}{*}{ Test } & \multirow[b]{2}{*}{$\begin{array}{l}\text { P- } \\
\text { value }\end{array}$} \\
\hline & \multicolumn{2}{|c|}{$\begin{array}{l}A \\
(\mathrm{~N}=42)\end{array}$} & \multicolumn{2}{|c|}{$\begin{array}{l}B \\
(\mathrm{~N}=42)\end{array}$} & & \\
\hline $\begin{array}{l}\text { Age }(Y) \\
\text { Mean } \pm \text { SD }\end{array}$ & \multicolumn{2}{|c|}{$23.66 \pm 2.62$} & \multicolumn{2}{|c|}{$23.47 \pm 2.73$} & $t=0.32$ & 0.190 \\
\hline $\begin{array}{l}\text { Parity: No, \% } \\
\text { P0 } \\
\text { P1 } \\
\text { P2 }\end{array}$ & $\begin{array}{l}18 \\
11 \\
13\end{array}$ & $\begin{array}{l}42.9 \\
26.2 \\
31.0 \\
\end{array}$ & $\begin{array}{l}18 \\
12 \\
12\end{array}$ & $\begin{array}{l}42.9 \\
28.6 \\
28.6 \\
\end{array}$ & $\chi^{2}=0.08$ & 0.959 \\
\hline $\begin{array}{l}\text { Basal TLC } \\
\text { Mean } \pm \text { SD }\end{array}$ & \multicolumn{2}{|c|}{$10.40 \pm 1.71$} & \multicolumn{2}{|c|}{$10.47 \pm 1.61$} & $t=0.19$ & 0.844 \\
\hline
\end{tabular}

Note: ${ }^{I}$ Total leucocytic count

Table2. Maternal Obstetric outcome of the studied groups

\begin{tabular}{|c|c|c|c|c|c|c|}
\hline \multirow{2}{*}{\begin{tabular}{|l|} 
\\
$\begin{array}{l}\text { Mode of delivery: No,\% } \\
\text { }{ }^{1} \text { SVD }\end{array}$
\end{tabular}} & \multicolumn{4}{|c|}{ Groups } & \multirow[b]{2}{*}{ Test } & \multirow[b]{2}{*}{$\begin{array}{l}\text { P- } \\
\text { Value }\end{array}$} \\
\hline & \multicolumn{2}{|c|}{$\begin{array}{l}A \\
(\mathrm{~N}=42)\end{array}$} & \multicolumn{2}{|c|}{$\begin{array}{l}\mathrm{B} \\
(\mathrm{N}=42)\end{array}$} & & \\
\hline${ }^{2} \mathrm{CS}$ & $\begin{array}{l}30 \\
12 \\
\end{array}$ & $\begin{array}{l}71.4 \\
28.6\end{array}$ & $\begin{array}{l}36 \\
6\end{array}$ & $\begin{array}{l}85.7 \\
14.3\end{array}$ & $\chi^{2}=2.54$ & 0.111 \\
\hline${ }^{3}$ PPH: No, \% & 3 & 7.1 & 3 & 7.1 & - & - \\
\hline Puerperal pyrexia: No, $\%$ & 6 & 14.3 & 5 & 11.9 & $\chi^{2}=0.10$ & 0.746 \\
\hline $\begin{array}{l}\text { Follow up TLC } \\
\text { Mean } \pm \text { SD }\end{array}$ & \multicolumn{2}{|c|}{$11.40 \pm 1.93$} & \multicolumn{2}{|c|}{$12.35 \pm 1.96$} & $\mathrm{t}=2.23$ & $0.028(S)$ \\
\hline
\end{tabular}

Note: ${ }^{1}$ Spontaneous vaginal delivery, ${ }^{2}$ Cesarean section, ${ }^{3}$ Postpartum hemorrhage

Table3. Neonatal outcome of the studied groups:

\begin{tabular}{|c|c|c|c|c|c|c|}
\hline & \multicolumn{4}{|c|}{ Groups } & \multirow[b]{2}{*}{ Test } & \multirow[b]{2}{*}{$\begin{array}{l}\text { P- } \\
\text { Value }\end{array}$} \\
\hline & \multicolumn{2}{|c|}{$\begin{array}{l}A \\
(\mathrm{~N}=42)\end{array}$} & \multicolumn{2}{|c|}{$\begin{array}{l}B \\
(\mathrm{~N}=42)\end{array}$} & & \\
\hline $\begin{array}{l}\text { Birthweight (gm) } \\
\text { Mean } \pm \text { SD }\end{array}$ & \multicolumn{2}{|c|}{$2461.90 \pm 136.95$} & \multicolumn{2}{|c|}{$2998.80 \pm 90.04$} & $t=21.22$ & $<0.001(\mathrm{HS})$ \\
\hline $\begin{array}{l}\text { APGAR } \\
\text { Mean } \pm \text { SD }\end{array}$ & \multicolumn{2}{|c|}{$8.16 \pm 0.76$} & \multicolumn{2}{|c|}{$8.35 \pm 0.72$} & $t=1.17$ & 0.245 \\
\hline${ }^{1}$ RDS: No,\% & 1 & 2.4 & $\mathbf{0}$ & 0.0 & $\begin{array}{l}\text { Fisher's } \\
\text { exact }=1.01\end{array}$ & 1.0 \\
\hline${ }^{2}$ TTN: No, $\%$ & 11 & 26.2 & 3 & 7.1 & $\chi^{2}=5.48$ & $0.019(S)$ \\
\hline${ }^{3}$ NICU: No,\% & 2 & 4.8 & 2 & 4.8 & - & - \\
\hline $\begin{array}{l}\text { Neonatal hyperbilirubinemia: } \\
\text { No, } \%\end{array}$ & 12 & 28.6 & 5 & 11.9 & $\chi^{2}=3.61$ & 0.057 \\
\hline
\end{tabular}

Note: ${ }^{1}$ Respiratory distress syndrome, ${ }^{2}$ Transient tachypnea of newborn, ${ }^{3}$ Neonatal intensive care unit 


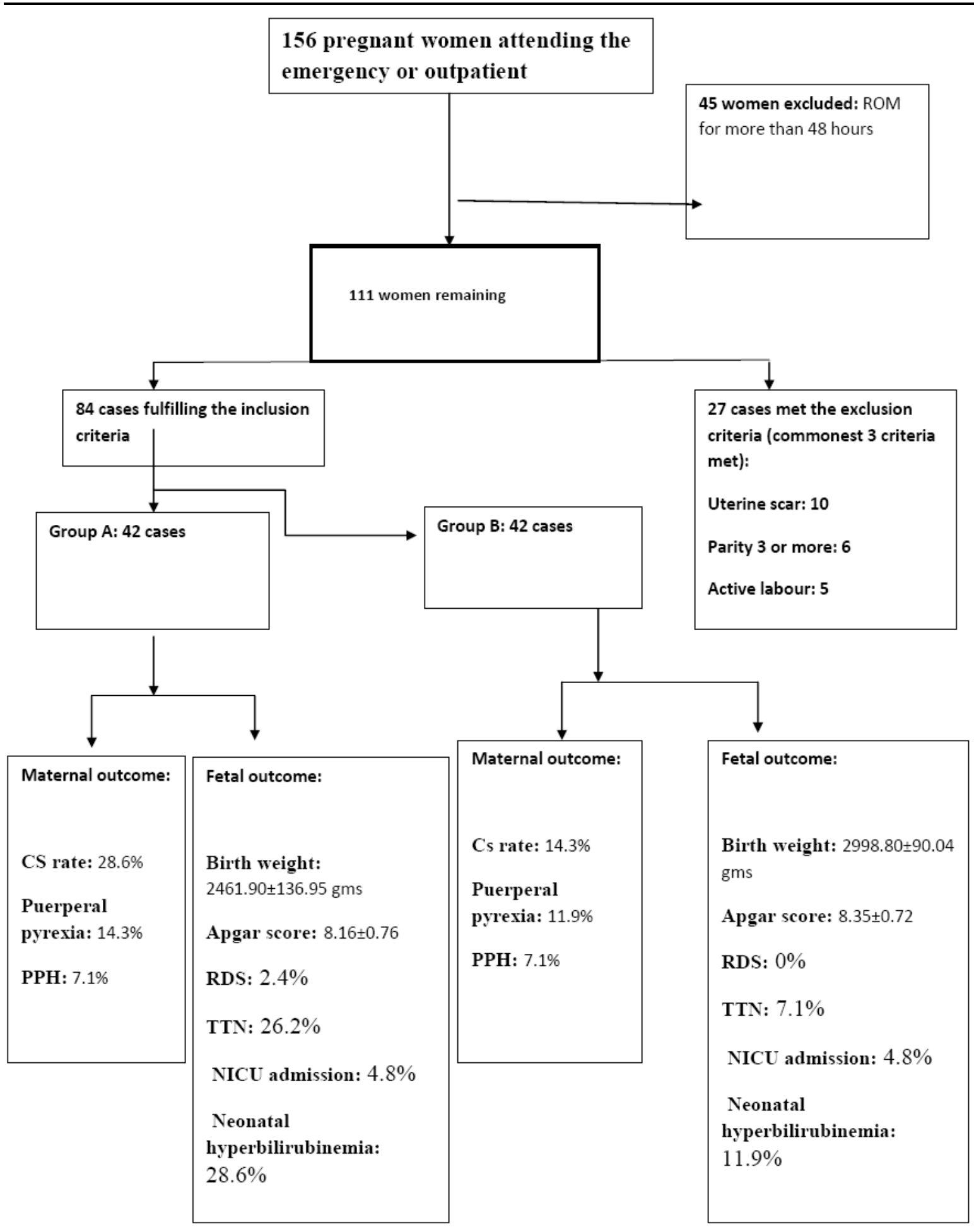

Figure1. Shows the flowchart diagram of the study women

- Group A refers to delivery group, group B refers to expectant group.

- ROM: rupture of membranes.

- CS: cesarean section.

- PPH: postpartum hemorrhage.

- gms: grams.

- RDS: respiratory distress syndrome.

- TTN: transient tachypnea of newborn.

- NICU: neonatal intensive care unit. 


\section{DISCUSSION}

Being responsible for about one third of preterm deliveries [9], which is the leading cause of neonatal morbidity and mortality, many efforts are done to advocate optimal management for preterm premature rupture of membranes. Regarding preterm delivery per se, without any associated complications, it is well known that term deliveries is much better than even late preterm deliveries regarding neonatal morbidity and mortality $[10,11]$ and for preterm deliveries, it is better when approaching term than earlier deliveries. But when there is preterm premature rupture of membranes, we are in a challenge between early delivery with hazards of prematurity and waiting with possible risks of maternal and fetal infection.

This study shew that, provided that, there is good antibiotic cover for cases of recent preterm premature rupture of membranes at 34 weeks of gestation, it seems to be better to manage expectantly for a reasonable period with a maximum of 2 weeks and this will reduce CS rate and puerperal endometritis, although not reaching statistical significance, and for neonatal outcome expectant management led to increase in birthweight and reduced the incidence of minor morbidities in the form of transient tachypnea of newborn and neonatal hyperbilirubinemia without any significant difference in major neonatal morbidity, and fortunately, there was no any mortality in both groups of our study.

Available data suggest that neonatal minor morbidity such as hyperbilirubinaemia and transient tachypnoea of the newborn was significantly higher among pregnancies delivered at 34 weeks of gestation or less compared with those delivered at 36 weeks [5]. Another study showsthat, although the incidence of RDS beyond 34 weeks was relatively low, the incidence of the affected infants was reduced with delaying delivery into the 36th week, with incidences of $10.4 \%$ and $1.5 \%$ at 35 and 36 weeks, respectively [12]. Another one [13]also suggests that neonatal morbidity and mortality is significantly reduced when delivery is achieved at 37 weeks compared to delivery at 34-36 weeks of gestation [13].

On 2010, the Cochrane database systematic review regarding the comparison of Planned early delivery to expectant management for cases of preterm premature rupture of membranes before 37 weeks' gestation found that there was no difference in the overall neonatal morbididity, including cerebroventricular haemorrhage, necrotizing enterocolitis, or duration of neonatal hospitalization and mortality between early and late deliveries, however, in early deliveries, there was increased maternal endometritis and cesarean section rate and this is consistent with that study[14].

It should be noted that incubator admission, even for minor disorders, is associated with maternal worrines because of iatrogenic morbidities which may be fatal that are reported in our developing country.

There is agreement between the results of our study and some authors $[5,15,16]$, although different designs and methodology, we are in agree that, provided that there is a good guard against infection, delivery at 36 weeks is better than delivery at 34 weeks for cases of preterm premature rupture of membranes.

On the other hand there are studies that did not conclude whether delivery or waiting is better in cases of near term preterm premature rupture of membranes[4, 14].

Also there are other studies supporting the decision of delivery for any case of rupture of membranes when gestational age reaches 34 weeks $[9,17,18]$.

There is still no obvious conclusion for the optimal management of those cases and from this study we can conclude that, provided that, the rupture of fetal membranes is recent and there is good antibiotic cover, it is wrranted to manage expectantly for a maximal of 2 weeks to decrease neonatal morbidity and incubator admission and its unsatisfactory outcomes.

The strength of this study resides in being prospective single center study with standardized management of all participants and a uniform protocol of neonatal resuscitation and care at NICU department.

Inability to design a double blind randomized trial and the small number of our patients were the main limitations of this study.

Future research should explore maternal acceptability and cost effectiveness of active versus expectant management in this population in a multicenter trial, in addition to the obstetric outcome. 


\section{ACKNOWLEDGEMENT}

The author would like to acknowledge the contribution of the residents and nursing staff of the labor and delivery ward of Menoufia university Hospital.

\section{Disclosure}

I certify that no actual or potential conflicts of interest in relation to this article exist.

\section{Authors' contribution to the manuscript}

E. Elshamy: Project development, Data Collection and Manuscript writing.

\section{REFERENCES}

[1] Parry S, Strauss $3^{\text {rd }}$ JF (1998). Premature rupture of the fetal membranes. N Engl U Med;338: 663-670.

[2] Farooqi A, Holmgren PA, Engberg S, Screnius F (1998). Survival and 2- year outcome with expectant management of second-trimester rupture of membranes. Obstet Gynecol; 92: 895-901.

[3] Falk SJ, Campbell LJ, Lee-parritz A et al (2004). Expectant management in spontaneous preterm premature rupture of membranes between 14 and 24 weeks gestation. J perinatol; 24: 611-616.

[4] Morris JM, Roberts CL, Crowther CA, Buchanan SL, Henderson-Smart DJ, Salkeld G (2006).protocol for the immediate delivery versus care of women with preterm prelabour rupture of membranes close to term (PPROMT) Trial[ISRCTN44485060]. BMC Pregnancy Childbirth; 6: 9.

[5] Lieman JM, Brumfield CG,CarloW, Ramsey PS (2005). Preterm premature rupture of membranes: is there an optimal gestational age for delivery? Obstet Gynecol;105:12-7.

[6] Msall ME, Tremont MR (2002). measuring functional outcomes after prematurity: developmental impact of very low birth weight and extremely low birth weight status on childhood disability. Ment Retard Dev Disabil Res Rev; 8: 258-272.

[7] Tarnow-Mordi W, Issacs D, Smart DH et al (2005). Neurodevelopemental impairement and neonatal infections. JAMA; 293: 932.

[8] Kenyon S, Boulvain M, Neilson J (2003). Antibiotics for preterm rupture of membranes. Cochrane Database Syst Rev;(2):CD001058.

[9] Mercer BM (2005). Preterm premature rupture of the membranes: current approaches to evaluation and management. Obstet Gynecol Clin N Am;32:411-28.

[10] Tomashek KM, Shapiro-Mendoza CK, Davidoff MJ, Petrini JR (2007). Differences in mortality between late-preterm and term singleton infants in the United States, 1995-2002. J Pediatr; $151: 450-6$.

[11] McIntire DD, Leveno KJ (2008). Neonatal mortality and morbidity rates in late preterm births compared with births at term. Obstet Gynecol; 111:35-41.

[12] Neerhof MG,Cravello C,Haney EI, Silver RK (1999).Timing of labor induction after premature rupture of membranes between 32 and 36 weeks' gestation.Am J Obstet Gynecol;180:349-52.

[13] Hauth JC(2006)Spontaneous preterm labor and premature rupture membranes at late preterm gestations: to deliver or not todeliver.Semin Perinatol.;30(2):98-102.

[14] Buchanan SL,Crowther CA, Levett KM,Middleton P,Morris J (2010). Planned early birth versus expectant management for women with preterm prelabour rupture of membranes prior to 37 weeks' gestation for improving pregnancy outcome. Cochrane Database Syst Rev; (3): CD004735.

[15] Kenyon S, Boulvain M, Neilson J (2004). Antibiotics for preterm rupture of the membranes: a systematic review. Obstet Gynecol;104:1051-7.

[16] Lim J, Allen V, Scott H, Allen A (2010). Late Preterm Delivery in Women With Preterm Prelabour Rupture of Membranes. J Obstet Gynaecol Can; 32(6): 555-560.

[17] ACOG Committee on Practice Bulletins-Obstetrics (2007). ACOG practice bulletin no. 80: premature rupture of membranes. Obstet Gynecol;109:1007-18.

[18] Pasquier J-C, Picaud J-C, Rabilloud M et al (2009). Neonatal outcomes after elective delivery management of preterm premature rupture of the membranes before 34 weeks gestation (DOMINOS Study). Eur J Obstet Gynecol Reprod Biol;143:18-23. 